This is a post-peer-review, pre-copyedit version of the article LongRange Radio for Underground Sensors in Geothermal Energy Systems published in Applications in Electronics Pervading Industry, Environment and Society APPLEPIES 2016.

The final authenticated version is available online at: http://dx.doi.org/10.1007/978-3-319-55071-8 1

Link at the published version: https://link.springer.com/chapter/10.1007\%2F978-3-319-55071-8_1 


\title{
Long-range Radio for Underground Sensors in Geothermal Energy Systems
}

\author{
Davide Brunelli ${ }^{1}$, Emanuele Bedeschi ${ }^{2}$, Marco Ferrari ${ }^{3}$, \\ Francesco Tinti ${ }^{3}$, Alberto Barbaresi ${ }^{4}$, Luca Benini ${ }^{2,5}$ \\ ${ }^{1}$ DII, University of Trento, 38123 Trento, Italy. \\ ${ }^{2}$ DEI, University of Bologna, 40136 Bologna, Italy. \\ ${ }^{3}$ DICAM, University of Bologna, 40136 Bologna, Italy. \\ ${ }^{4}$ DipSA, University of Bologna, 40127 Bologna, Italy. \\ ${ }^{5}$ IIS, ETHz, 8092 Zurich, Switzerland.
}

\begin{abstract}
The paper presents the design of a temperature monitoring system in a very harsh environment, such as Shallow Geothermal Systems (SGS), where the information of underground temperature is necessary to assess the thermal potential of the soil, for maximizing the efficiency of the SGS. The challenge is to get information at different depths (sometimes up to - $100 \mathrm{~m}$ ), to transmit data wirelessly in rural areas where conventional wireless connections (e.g. WiFi, GSM) are not guaranteed and energy availability poses severe limits. Our design exploits a recent new modulation protocol developed for long-range transmission, at the minimum energy cost, and a two-tier hardware architecture for measuring underground temperature. Aggressive duty cycling permits to achieve lifetime of several years. Experimental results demonstrate the utility of such a system during the design and the operational activity of a SGS.
\end{abstract}

\section{Introduction}

In recent years, energy saving in several industrial and agricultural areas, such as food and pharmaceutical industries, became an important topic [1]. Geothermal systems are among the most promising technologies to contain heating and air conditioning consumptions and have proved to be an effective solution to reduce the electrical bill, especially in sectors where air conditioning for large spaces or tight temperature control is necessary, such as cold-chain storage for food production or distribution, and cold biopharma warehouses [2]. In such systems, accurate, long-term underground measurements are essential, because the temperatures in the upper soil's layers show significant daily and annual fluctuations and the correct knowledge of the subsoil state drives the control of the geothermal system. The heat flow below the ground level is influenced by several parameters, such as air temperature, wind speed, season, shading/irradiation, soil thermal properties, which are characterized by irregular variability. Therefore, subsoil temperature estimation is often a hard task, in particular within the layers at few meters of depth, where the influence factors are multiple [3] and where the seasons and air temperature play a crucial role, as seen in Fig. 1; where each month of the year shapes differently the profile of the average temperatures at various depths.

The use of fiber cables thermometers in the shallow geothermal measurements 
projects has been applied since many years [4], while many other applications preferred the use of wireless sensors installed in the ground or within the boreholes [5]. Unfortunately, most of them have short transmission range, which limit their usage because they are mainly variations of previous projects developed for agriculture. For example, some Wireless Sensor Networks (WSN) permit to measure and to control applications in viticulture [6], large farms [7] and other plants [8], but radio technology is not suitable for underground measurements, unless using very high power for transmissions, in the order of Watts [3], hardly compatibles with lowpower system and long-life constraints.

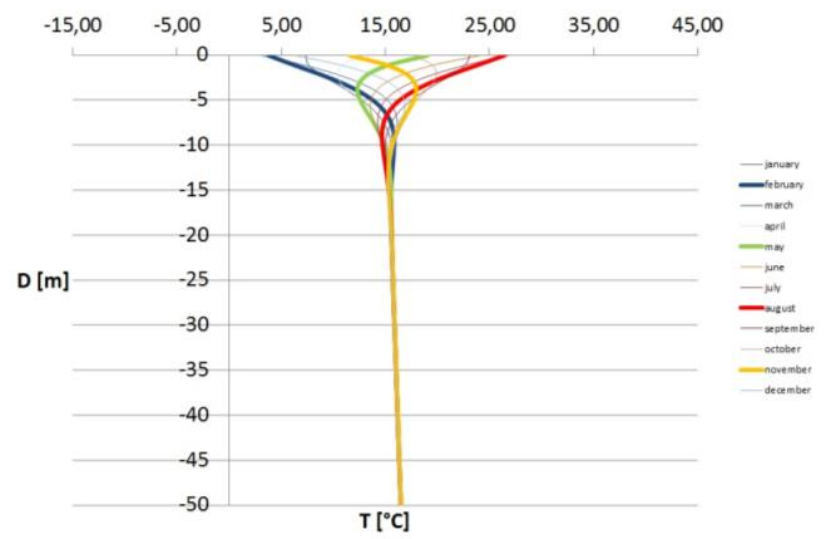

Fig. 1. Graphical representation of the simplified model for the underground temperature evolution along depth for a specific site.

We present a novel, inexpensive and robust monitoring system for long-term underground temperature measurements. This apparatus consists in a set of sensors capable to acquire, to process and to transmit measured temperatures at different depths and on different humidity conditions, with the accuracy and time constraints typical of the industrial control applications. In detail, the SGS underground area contains a cylindrical basket heat exchanger of $1.5 \mathrm{~m}$ high and $0.6 \mathrm{~m}$ radius, deployed as shown in Fig. 2.

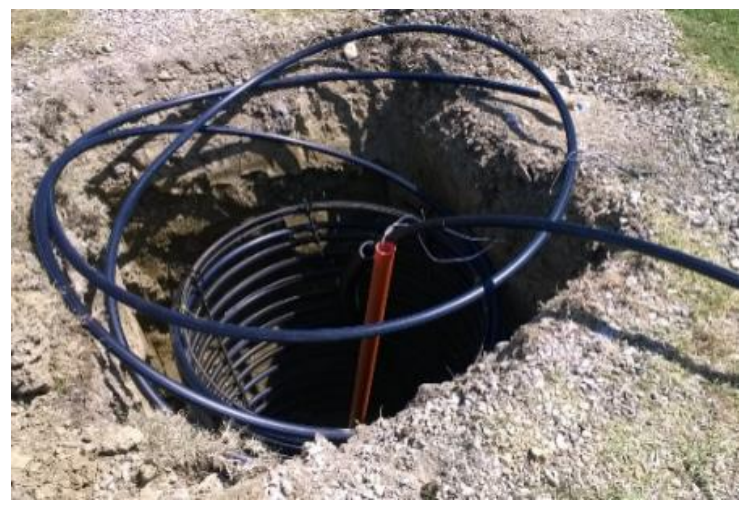

Fig. 2. The installation of the buried heat exchanger with the shape of a cylindrical basket whose performance is influenced by thermal properties of the soil. 
Heat transfer is evaluated by measuring how subsoil layers temperature changes over time. Required depths for the measurements are $-2 \mathrm{~m},-4 \mathrm{~m}$ and $-6 \mathrm{~m}$, at a distance of $1.4 \mathrm{~m}$ from the geothermal basket, used as heat exchanger, and around $8 \mathrm{~m}$ from the Thermal Response Test machine (TM) as illustrated in Fig. 3. A micro-Thermal Response Test (UTRT) machine has been designed to assess the thermal resistance of the soil and the performance of a SGS in [9].

\section{System Architecture}

We used an innovative hybrid approach to address the challenges of underground sensing, mixing wired connectivity to the deep sensors and wireless long-range, lowpower connectivity for the near-surface units to the main gateway. A series of devices designed with a temperature sensor are placed underground, through specific boreholes, called Depth Board (DB), as illustrated in Fig.3. These are wired connected to another board, called Surface Board (SB), positioned few centimeters below the ground level and repaired inside a concrete manhole. Classic radio technologies such as WIFI or GSM are too much expensive and energy-hungry for this context. Even other traditional sub-GHz radio technologies in underground WSN [4] need too much power for the required system lifetime. Thus, we used a recent technology based on Long-Range (LoRa) wireless transmission (at sub-GHz frequency) that allows low-power connectivity with some $\mathrm{km}$ range for the nearsurface units. Moreover, aggressive application duty cycle permits to achieve multiyear lifetime when battery powered. The SBs are organized in a star-topology, and transmit wirelessly the acquired data to a gateway $(\mathrm{GW})$, which is the collection point for the WSN. The connection is peer-to-peer without the need of a complex MAC protocol. The GW aggregates and forwards the information to the Cloud through an internet connection (e.g. GSM).

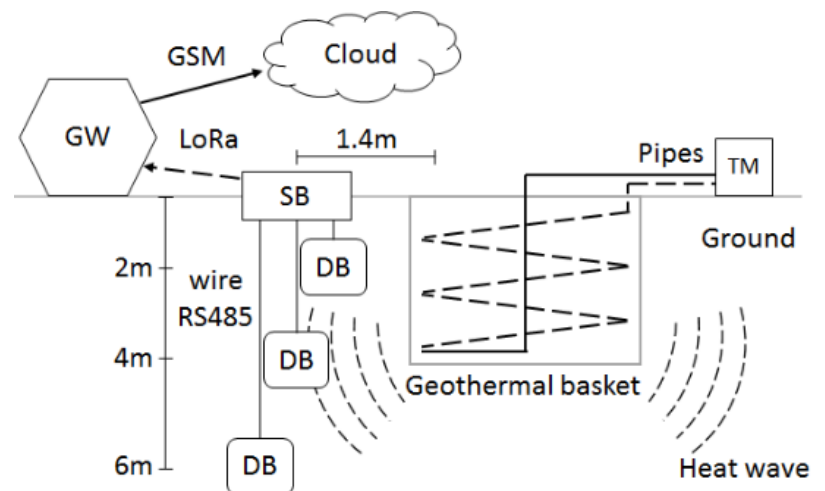

Fig. 3. The model (not to scale) of the area where underground monitoring is necessary for a SGS.

\subsection{Depth node (DB)}

This board is manufactured to fit a sealed container and to be powered from the surface through a cable that carries RS485 communication signals. RS485 is used for 
wired connections to a SB node, and each surface node can manage up to 3 DB nodes. The depth-node is subject to power gating, in fact it can be completely switched off when measuring underground temperature is not necessary, as illustrated in Fig.3. The sensor SHT21 (manufactured by Sensirion) has been mounted to measure the temperature with one hundredth of a degree accuracy. The TI MSP430FR5969 microcontroller features low power consumption while RS485 communication is realized using a Maxim MAX3485 transceivers to achieve a transmission distance of up to $100 \mathrm{~m}$.

\subsection{Surface Board (SB)}

The main characteristic of these boards, shown in Fig.4, is the exploitation of a Long-Range radio transceiver at $868 \mathrm{MHz}$. With the aim of low power consumption, the same low-power MCU is used, TI MSP430FR5969, equipped by a Real Time Clock for synchronizing the tasks to execute and the transmissions to the gateway. Wireless communication to the gateway is realized using LoRa radio technology provided by Semtech [10], operating at $868 \mathrm{MHz}$.

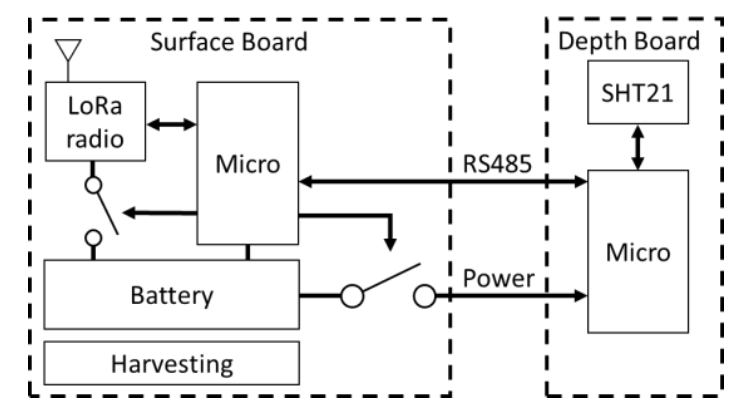

Fig. 4. Architecture of multi-module sensor node with power gating.

LoRa, takes advantage of a proprietary modulation technique based on the spreading spectrum, that gives it a strong noise immunity and thanks to a $-148 \mathrm{~dB}$ high sensitivity, allows reaching long distances with a very low power consumption. In addition, we added a boost power amplifier that can transmit up to $+20 \mathrm{~dB}$ power, which is the maximum allowed in industrial applications and permits a transmission range of several kilometers.

The radio parameters are adjusted for the optimal trade-off between power consumption and transmission range, after dimensioning the bandwidth and the spreading factor. We set a payload of 13 bytes, and a symbol time $\mathrm{T}_{\mathrm{s}}=8.19 \mathrm{~ms}$. Therefore, according to the datasheet, $T_{\text {packet }}=247.81 \mathrm{~ms}$ the total time-on-air of a packet. We configured $+2 \mathrm{~dB}$ for signal output power that is sufficient to cover the distance between sensors and the gateway in our case and in any weather condition, we measured a $38 \mathrm{~mA}$ for as current consumption during the interval time $\mathrm{T}_{\text {packet. }}$. This accounts for about $31 \mathrm{~mJ}$ in the total energy budget. Further improvements can be achieved using aggressive data compression techniques as proposed in [11]-[13]

The nodes are equipped with a lithium polymer battery of $1000 \mathrm{mAh}$, and mounts a high efficiency DC/DC based on TI BQ25570, which can ensure multi-year long 
lifetime to the system and host also energy harvesting modules (see [14][15]). Since the radio transceiver is the most energy hungry component, we have implemented a power gating mechanism, using a MOSFET to enable the transceiver supply when needed and to achieve zero power consumption, when communication is off. The prototype of the Surface Board is shown in Fig. 5.

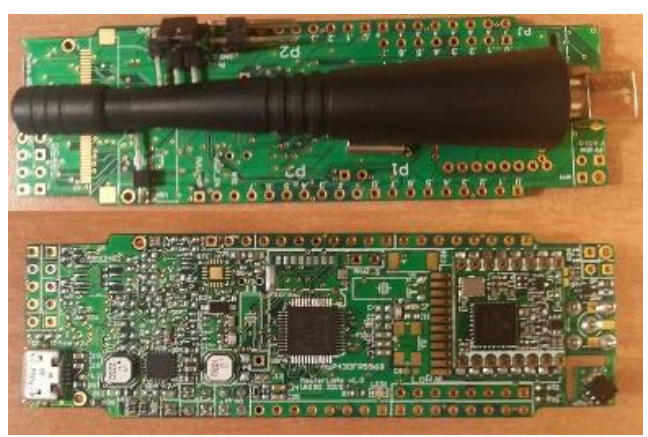

Fig. 5. Prototypes of the wireless module with LoRa transceiver.

\section{Experimental Results}

The power consumption profile is presented in Fig. 6. The peak of current consumption is required when transmitting the information and may reach about $38 \mathrm{~mA}$. During the sleep interval, the power consumption cut down to few $\mu \mathrm{W}$, which is enough to guaranteed adequate operating time. Typical sending intervals are in the order of 15 minutes or hours.

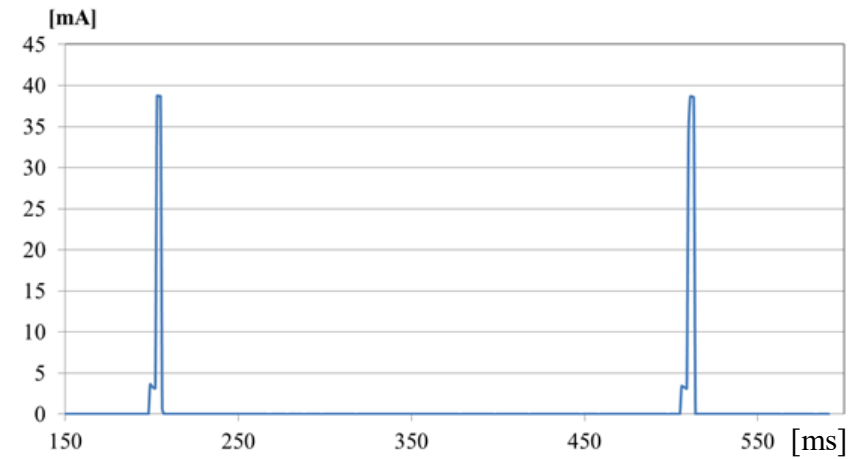

Fig. 6. Power profile of the SB during operation.

In detail, after a first initialization of the peripherals and the clock, where the consumption can be neglected considering that it occurs only at the beginning, the breakdown of the power consumption can be modeled as in Fig. 7. Every cycle can be split into three intervals: sensing/processing interval $\left(T_{s p}\right)$, transmission interval $\left(T_{t x}\right)$ and sleep interval in ultralow power mode $\left(T_{\text {lowp }}\right)$. We measured $T_{s p}=0.3 \mathrm{~s}$ and an 
average current consumption of $\mathrm{I}_{\mathrm{sp}}=3 \mathrm{~mA}$, which results in an energy of $2.97 \mathrm{~mJ}$ for each DB connected.

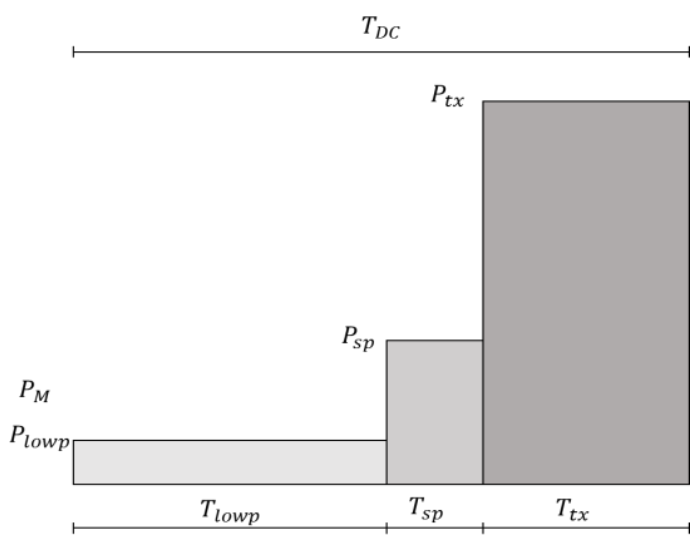

Fig. 7. Power profile model of the SB.

When the system is in low-power mode, during sleep interval, the total system consumption drops down to $\mathrm{I}_{\mathrm{low}}=3 \mu \mathrm{A}$, because only the RTC is powered. Considering that the measuring rate for such kind of applications is about $\mathrm{T}_{\text {meas }}=15 \mathrm{~min}$, the application runs with a Duty-cycle $\mathrm{D}=0.06 \%$ and the average power consumption squeezes to $47.6 \mu \mathrm{W}$ Therefore, the operating lifetime of the system, if powered with a lithium polymer battery of $1000 \mathrm{mAh}$ and all the three DB are connected, is estimated in $\mathrm{T}_{\text {life }}=3.1$ years.

Fig. 8 shows an image of the real deployment used to perform the tests. The surface board is clearly visible connected to the cables to three depth nodes.

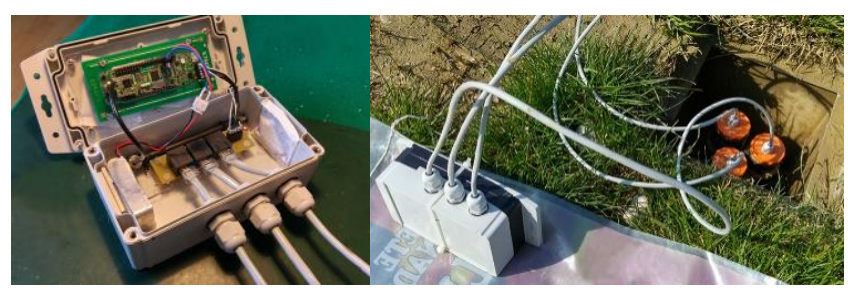

Fig. 8. Prototype of the long-range radio module.

The trend of the temperature measurement and an example of usage is depicted in Fig. 9, where temperature oscillations at the depth of $-2 \mathrm{~m}$ (blue), $-4 \mathrm{~m}$ (orange) and $6 \mathrm{~m}$ (green) are provided. A deeper analysis of the curves indicates how underground thermal diffusivity influences the heat transfer velocity at different depths and distances from the basket. 


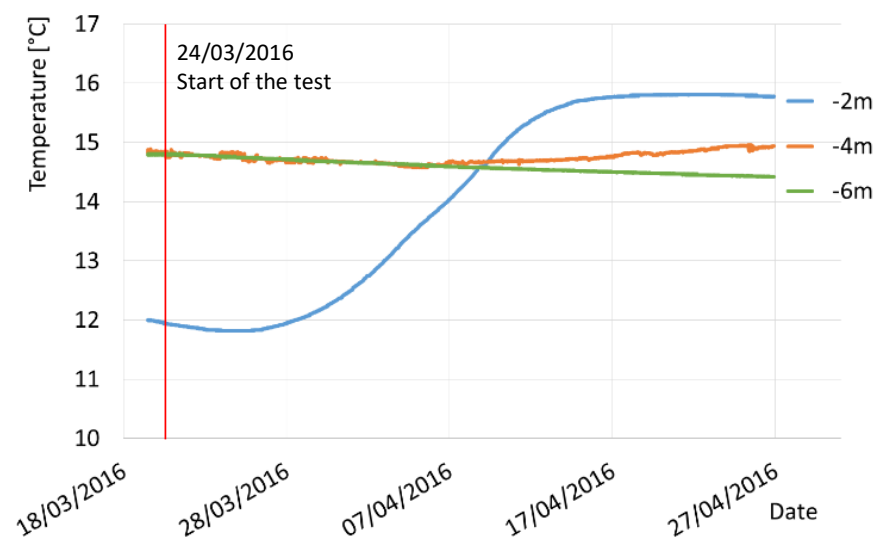

Fig. 9. Plot of underground temperature inside the borehole, at $2 \mathrm{~m}, 4 \mathrm{~m}$ and $6 \mathrm{~m}$ depth.

In fact, we performed more long-duration tests, started on March $24^{\text {th }}$, 2016 with the injection of hot water into the geothermal basket. The test was conducted uninterruptedly for 12 days, with a constant water flow of $800 \mathrm{l} / \mathrm{h}$ and a total electric resistance powered constantly with $1500 \mathrm{~W}$, power was used to heat the water at the inlet of the basket. Then we kept the water circulating into the basket without the heating resistance, for a release period of additional 12 days. The heat wave arrived after 4 days at $2 \mathrm{~m}$ depth and $1.4 \mathrm{~m}$ far from the geothermal basket, and after 11 days at $4 \mathrm{~m}$ depth; while it never reached $6 \mathrm{~m}$ depth in the considered period. We note that this is extremely valuable information for the experts asked to design the geothermal system, because these measurements indicate that there is no need of large spacing among multiple baskets, in the considered test site. In addition to the initial test phase, this sensor deployment will be used in long-term measurements after the construction of the SGS system, because underground real-time temperatures will drive the geothermal control system, according to specific temperature damping model.

\section{Conclusion}

We discussed the design of an autonomous wireless monitoring system for underground temperatures. A complete set of sensors designed to operate several meters below ground level, was designed and deployed to understand the external factors influencing the behavior of the soil when a geothermal system is to be installed. Design challenges such as battery lifetime and radio transmission over long distances at the lowest power consumption have been addressed. The designed boards are capable to operate unattended for at least 3 years, sending information to a gateway located several km away.

\section{Acknowledgements}

The research contribution presented in this paper has been supported by the EuroCPS Project (grant n. 644090) and by the FLEXMETER Project (grant n.646568), both funded by the EU H2020. Moreover, the authors would like to thank 
"AziendaBranchini" for letting us installing the prototype and all the instrumentation measurements on site for our experimental tests.

\section{References}

[1] A. Barbaresi, D. Torreggiani, S. Benni, et al. Underground cellar thermal simulation: Definition of a method for modelling performance assessment based on experimental calibration. Energy Build. 2014; 76:363-372

[2] B. Sanner, C. Karytsas, D. Mendrinos, L. Rybach Current status of ground source heat pumps and underground thermal energy storage in Europe (2003) Geothermics, 32 (2003), pp. 579-588

[3] Ian F. Akyildiz, Erich P. Stuntebeck (2006), "Wireless underground sensor networks: Research challenges" Broadband and Wireless Networking Laboratory, School of Electrical and Computer Engineering, Georgia Institute of Technology, 75 5th St. NW, Atlanta, GA 30308, USA

[4] H. Fujii, H. Okubo, K. Nishi, R. Itoi, K. Ohyama, K. Shibata (2009) "An improved thermal response test for U-tube ground heat exchanger based on optical fiber thermometers", Geothermics, Volume 38, Issue 4, December 2009, Pages 399-406.

[5] J. Martos, A. Montero, J. Torres, J. Soret, G. Martinez, R. Garcio-Olcina "Novel Wireless Sensor System for Dynamic Characterization of Borehole Heat Exchangers" Sensors 2011, 11, 7082-7094.

[6] A. Matese, S.F. Di Gennaro, A. Zaldei, L. Genesio, F.P. Vaccari, A wireless sensor network for precision viticulture: The NAV system, Computers and Electronics in Agriculture, Volume 69, Issue 1, November 2009, Pages 51-58, ISSN 0168-1699

[7] R. Beckwith, D. Teibel, and P. Bowen. 2004. Report from the Field: Results from an Agricultural Wireless Sensor Network. In Proc. of the 29th Annual IEEE International Conference on Local Computer Networks (LCN '04). IEEE Computer Society, USA, 471-478

[8] T. Ojha, S. Misra, and N. S. Raghuwanshi. 2015. Wireless sensor networks for agriculture. Comput. Electron. Agric. 118

[9] A. Verdecchia, D. Brunelli, F. Tinti, A. Barbaresi, P. Tassinari and L. Benini, "Low-cost micro-thermal response test system for characterizing very shallow geothermal energy," IEEE Workshop on Environmental, Energy, and Structural Monitoring Systems (EESMS), Bari, 2016, pp. 1-6.

[10] Semtech SX1276 "137 MHz to 1020 MHz Low Power Long Range Transceiver", http://www.semtech.com/wireless-rf/rf-transceivers/sx1276/

[11] C. Caione, D. Brunelli and L. Benini, "Compressive Sensing Optimization for Signal Ensembles in WSNs," in IEEE Transactions on Industrial Informatics, vol. 10, no. 1, pp. 382-392, Feb. 2014

[12] D. Brunelli.; C. Caione. Sparse Recovery Optimization in Wireless Sensor Networks with a Sub-Nyquist Sampling Rate. Sensors 2015, 15, 16654-16673

[13] B. Milosevic; C. Caione; E. Farella; D. Brunelli; L. Benini. Sub-Sampling Framework Comparison for Low-Power Data Gathering: A Comparative Analysis. Sensors 2015, 15, 5058-5080.

[14] D. Dondi, D. Brunelli, L. Benini, P. Pavan, A. Bertacchini and L. Larcher, "Photovoltaic cell modeling for solar energy powered sensor networks," Advances in Sensors and Interface, 2007. IWASI 2007. 2nd International Workshop on, Bari, 2007, pp. 1-6.

[15] C. Moser, L. Thiele, D. Brunelli and L. Benini, "Robust and Low Complexity Rate Control for Solar Powered Sensors," 2008 Design, Automation and Test in Europe, Munich, 2008, pp. 230-235. 